\title{
Stability of Temporally-Evolving Supersonic Boundary Layers over Micro-Cavities for Ultrasonic Absorptive Coatings
}

\author{
Guillaume A. Brès* and Tim Colonius ${ }^{\dagger}$ \\ California Institute of Technology, Pasadena, CA 91125, U.S.A. \\ Alexander V. Fedorov \\ Moscow Institute of Physics and Technology, Zhukovsky, Moscow region, 140180, Russia
}

Ultrasonic absorptive coatings, consisting of regularly-spaced arrays of micro-cavities, have previously been shown to effectively damp second-mode instability for the purpose of delaying transition in hypersonic boundary layers. However, previous simulations and stability analysis have used approximate porous-wall boundary conditions. Here we investigate the feasibility of using direct numerical simulation to directly compute the hypersonic boundary layer including the micro-cavities. In order to keep the problem computationally tractable, we restrict our attention to the two-dimensional case (which is relevant since the second-mode is initially two dimensional), and we show that temporally evolving layers display qualitatively similar behavior to spatially developing boundary layer and instabilities. We validate the numerical method by comparing the simulation results to temporal linear stability analysis of the (frozen) velocity and temperature profiles from the direct numerical simulation. Two-dimensional linear simulations of the boundary layer on a flat plate and over a porous coating are performed, and it is shown that the presence of the cavities attenuates the instability waves, as expected from theory.

\section{Nomenclature}

*Postdoctorate Fellow, MC 104-44, Dept. of Mechanical Engineering, Member AIAA

${ }^{\dagger}$ Professor, MC 104-44, Dept. of Mechanical Engineering, Member AIAA

${ }^{\ddagger}$ Professor, Dept. of Aeromechanics and Flight Engineering, Member AIAA 


$\begin{array}{ll}A r & \text { Cavity aspect ratio } \\ a & \text { Speed of sound } \\ b & \text { Cavity half-width } \\ f & \text { Frequency } \\ H & \text { Cavity depth } \\ M & \text { Mach number } \\ \operatorname{Pr} & \text { Prandtl number } \\ R e_{\delta^{*}} & \text { Reynolds number } \\ s & \text { Cavity spacing } \\ T & \text { Temperature } \\ t & \text { Time } \\ U & \text { Freestream streamwise velocity } \\ u & \text { Streamwise velocity } \\ v & \text { Normal velocity } \\ x & \text { Streamwise direction } \\ y & \text { Normal direction } \\ \tilde{\alpha} & \text { complex streamwise wavenumber } \\ \alpha & \text { streamwise wavenumber } \\ \tilde{\beta} & \text { complex spanwise wavenumber } \\ & \end{array}$

$\gamma \quad$ Specific heat ratio

$\delta \quad$ Boundary layer thickness

$\delta^{*} \quad$ Boundary layer displacement thickness

$\lambda \quad$ Streamwise wavelength

$\mu \quad$ Viscosity

$\rho \quad$ Density

$\sigma \quad$ growth rate

$\phi \quad$ Porosity

$\Omega \quad$ Complex Angular frequency

$\omega \quad$ Angular frequency

Subscript

$\infty \quad$ freestream quantity

$w \quad$ Property at the wall

$S \quad$ Spatial analysis property

$T$ Temporal analysis property

Superscript

- Mean (frozen) flow quantity

' Perturbed quantity

\section{Introduction}

For the next-generation of hypersonic vehicles, efficient laminar flow control (LFC) technologies that delay laminar-turbulent transition are required to reduce heat transfer rates and in turn, reduce the weight and complexity of thermal protection systems (TPS). Because of the high local Mach numbers and low wall temperature ratios on the vehicle surface, the second mode (or Mack mode) is the dominant instability causing laminar-turbulent transition (rather than the first mode associated with Tollmien-Schlichting waves). As the second mode represents high-frequency (ultrasonic) acoustic waves trapped in the boundary layer, a passive ultrasonic absorptive coating (UAC), which consists of a thin perforated layer of regular microstructure, has been shown to stabilize the second mode and dramatically increase laminar run. ${ }^{1,2}$ Additional experimental and theoretical studies ${ }^{3-6}$ have confirmed the robustness of the UAC stabilization concept and motived our present numerical effort to mature this LFC methodology and improve the UAC designs.

In order to perform direct numerical simulations (DNS) of the UAC, some tradeoffs are required in order to deduce a set of computationally tractable model problems, since a 
realistic surface would have as many as 20 pores per wavelength of the most unstable wave. ${ }^{3}$ In this work, we show that a simplified configuration that considers a temporally evolving boundary layer on an infinite porous plate retains enough of the relevant flow physics in order to meet the overall modeling objectives of this task. The temporally evolving boundary layer neglects the spatial growth of the boundary layer, and instead diffuses slowly with time. Over short time-scales associated with acoustic energy attenuation in UAC, the laminar boundary layer is essentially frozen, consistent with either a spatial or temporal description of the mean flow field, and consistent with parallel flow approximations that are typically made in instability calculations.

The temporal transformation is depicted in Figure 1. Spatial evolution (at left) would require that a large number of pores be considered in order to resolve the many wavelengths of instability wave that would be required to avoid entrance and downstream boundarycondition effects in typical computations. On the other hand, the detailed physics associated with UAC all occur over a single wavelength of the instability wave; in the temporal approximation at most 20 pores need be directly resolved with the computational mesh.

(a)

(b)

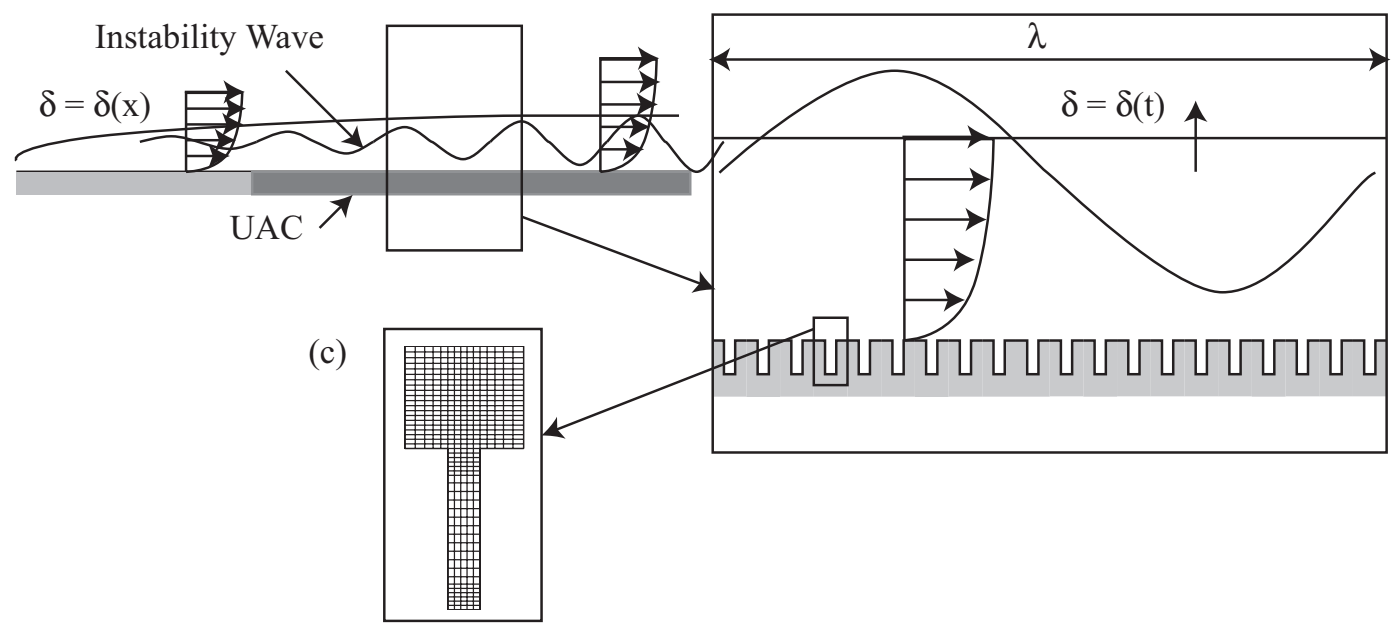

Figure 1. Schematic diagram for DNS of UAC (not to scale). (a) the spatial problem with many wavelengths of the spatially growing instability wave; (b) the temporal problem with a single wavelength of the temporally growing instability wave; (c) a portion of the computational grid around a single pore.

In this paper, we conduct numerical simulations with a standard no-slip wall in order to validate the use of the temporally-evolving assumption on both the mean hypersonic boundary layer and instability wave development. The existing spatially-evolving linear stability theory is reformulated to enable comparison with the present linear results and assess possible discrepancies in wave phase-speeds and growth rate. The numerical simulations are performed with constant fluid properties, while theoretical stability calculations are con- 
ducted with both constant and temperature-dependent properties. An additional benefit of the temporal simulation is that shocks are avoided during boundary layer growth, and linear and early nonlinear stages of instability growth, thus allowing us to use non-dissipative, high-order numerics. The simulations presented here, along with the simulations of the acoustic scattering of porous coatings presented in Ref. 7, bracket the flow physics for the full problem, and are sufficiently simple to allow a parametric study of the geometrical factors (pore size, aspect ratio, spacing) and flow conditions (free-stream velocity and temperature, boundary layer thickness, Reynolds number) relevant to UAC design.

\section{Temporal Stability of Compressible Boundary Layer}

\section{A. Linear Stability Theory}

The existing spatially-evolving linear stability theory for hypersonic boundary layer ${ }^{1}$ over flat plates is reformulated to enable comparison with the present temporally-evolving instability results. We assume that the flow field $\mathbf{q}=[\rho u, \rho v, \rho w, \rho, e]^{T}$ can be decomposed into $\mathbf{q}=$ $\overline{\mathbf{q}}+\mathbf{q}^{\prime}$, where $\overline{\mathbf{q}}$ is a mean flow and the perturbation field $\mathbf{q}^{\prime}$ verifies $\mathbf{q}^{\prime} \ll \overline{\mathbf{q}}$. The NavierStokes equations are then linearized about $\overline{\mathbf{q}}$ by neglecting higher-order terms in $\mathbf{q}^{\prime}$ to give a first-order approximation. Here, the mean flow is characterized by $\overline{\mathbf{q}}(y)$, based on the locally parallel approximation, and the three-dimensional perturbation field $\mathbf{q}^{\prime}$ is written as follow:

$$
\mathbf{q}^{\prime}(x, y, z, t)=\operatorname{Re}(\hat{\mathbf{q}}(y) \exp [i(\tilde{\alpha} x+\tilde{\beta} z-\Omega t)]),
$$

where $\tilde{\alpha}$ and $\tilde{\beta}$ are the nondimensionalized wavenumbers, and $\Omega$ is the angular frequency. Since the second mode is the dominant instability in two-dimensional and quasi-two-dimensional supersonic boundary layers, the parameter $\tilde{\beta}$ is set to 0 .

For spatial stability, $\Omega$ is taken to be a real and prescribed frequency $\omega_{S}$, and the eigenvalue problem is solved for the complex eigenvalue $\tilde{\alpha}$. If $\operatorname{Im}(\tilde{\alpha})<0$, then the flow is unstable with spatial growth rate $\sigma_{S}=-\operatorname{Im}(\tilde{\alpha})$ and streamwise wavenumber $\alpha_{S}=\operatorname{Re}(\tilde{\alpha})$.

In our case, we are interested in the temporal stability: $\tilde{\alpha}$ is a real and prescribed streamwise wavenumber $\alpha_{T}$, and $\Omega$ is the complex eigenvalue. If $\operatorname{Im}(\Omega)>0$, then the flow is unstable with temporal growth rate $\sigma_{T}=\operatorname{Im}(\Omega)$ and frequency $\omega_{T}=\operatorname{Re}(\Omega)$. Eventually, the determination of the least damped (or most unstable) modes for a given wavelength $\alpha_{T}$ amounts to finding the eigenvalue $\Omega$ and corresponding eigenvector $\hat{\mathbf{q}}$ by integrating the governing equations directly in the time domain.

To a first approximation (valid for weakly unstable modes), the spatial and temporal formulation can be related using Gaster transformation. In this approximation, $\Omega$ is assumed to be an analytic function of $\tilde{\alpha}$, and the following equations are obtained by integration of 
the Cauchy-Riemann relations, and neglecting higher order terms:

$$
\alpha_{S}=\alpha_{T}=\alpha, \quad \omega_{S}=\omega_{T}=\omega, \quad \sigma_{S}=\sigma_{T} / C_{g},
$$

where $C_{g}=\partial \omega_{T} / \partial \alpha_{T}$ is the group velocity. The transformation requires that the (numerical) data for the eigenvalues be differentiated.

\section{B. Spatial versus temporal stability}

First, we start with the typical linear analysis of a spatially developing compressible boundary layer, and show that the temporal formulation leads to similar results. The mean-flow compressible Blasius solutions are calculated in the locally parallel approximation for a flat plate at Mach $M=U / a_{\infty}=6$, for an ideal gas with $\gamma=1.4$ and $\operatorname{Pr}=0.7$. The temperatureviscosity dependences are Sutherland law (with constant $S=110.4 \mathrm{~K}$ and $T_{\infty}=330 \mathrm{~K}$ ) and power-law $\mu / \mu_{\infty}=\left(T / T_{\infty}\right)^{m}$, with $m=1$. The chosen length scale for the flow is the displacement thickness $\delta^{*}$. The Blasius profiles are self-similar, and do not depend on the Reynolds number. Comparison of these profiles is shown in figure 2 and show little variation for the different temperature-viscosity dependences considered. Therefore, the stability analysis is conducted only for the mean flow with the power-law for viscosity.

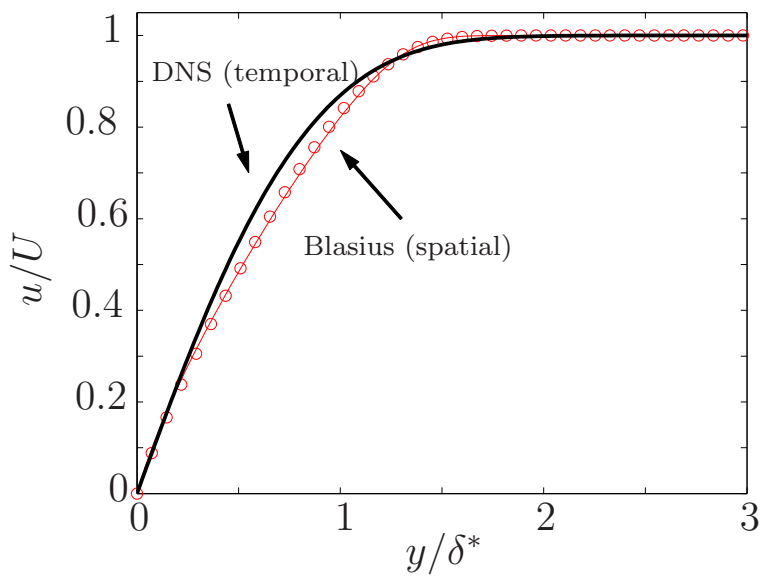

$(a)$

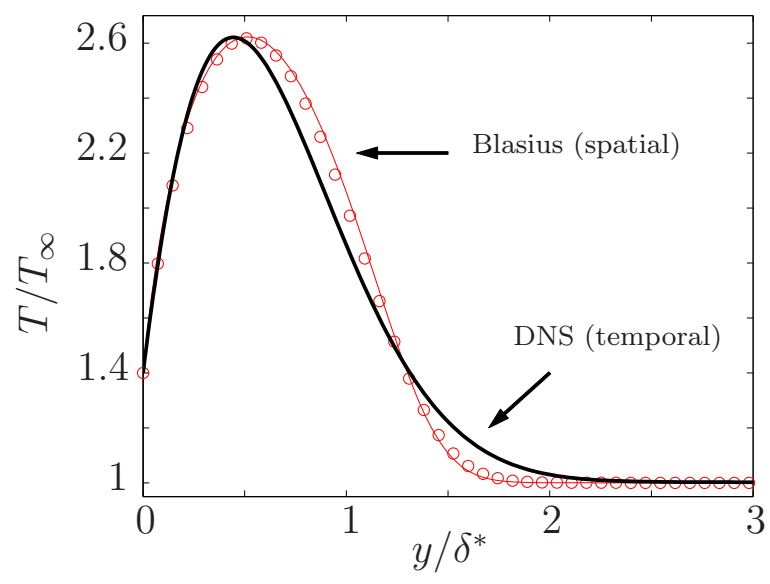

(b)

Figure 2. Comparison of the compressible boundary layer profiles from temporal DNS at $R e_{\delta^{*}}=4200$ with constant viscosity ( - ) , and from Blasius solution with temperatureviscosity dependence in Sutherland law ( $\circ)$, and in power-law ( $\_$). The parameters are $M=6, T_{w} / T_{\infty}=1.4$. (a) streamwise velocity; (b) temperature.

The theoretical stability calculation are computed for the two-dimensional second-mode waves at $R e_{\delta^{*}}=\rho_{\infty} U \delta^{*} / \mu_{\infty}=4200$, following the method described in Ref. 1 . These calculations can be conducted for any locally parallel profile. Here, both spatial and temporal 
analysis are performed using the Blasius profile in figure 2. The comparison is shown in figure 3, using the Gaster transformation. The results are in excellent agreement and demonstrate that the development of the second-mode instability is similar for spatially and temporally evolving boundary layer. Therefore, we argue here that the temporal formulation is appropriate to study UAC performance, and that results for practical applications with spatially developing boundary layer and instabilities can be obtained from our temporal DNS with the Gaster transformation.

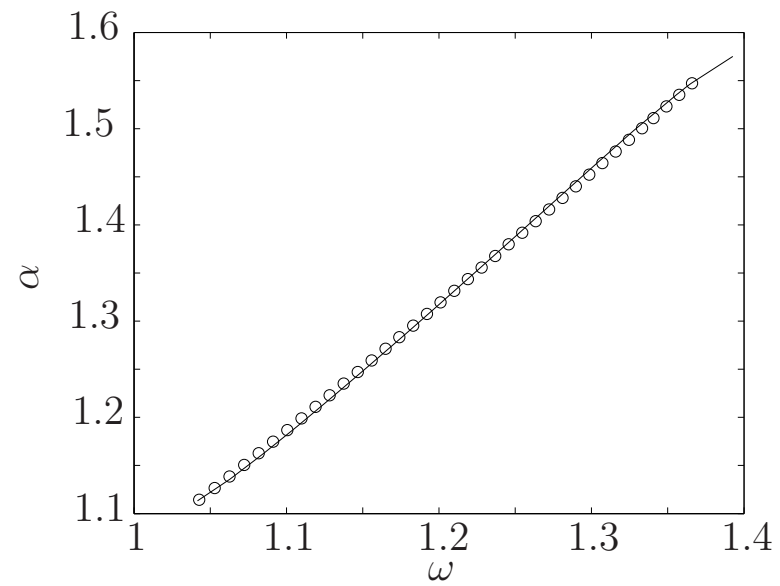

(a)

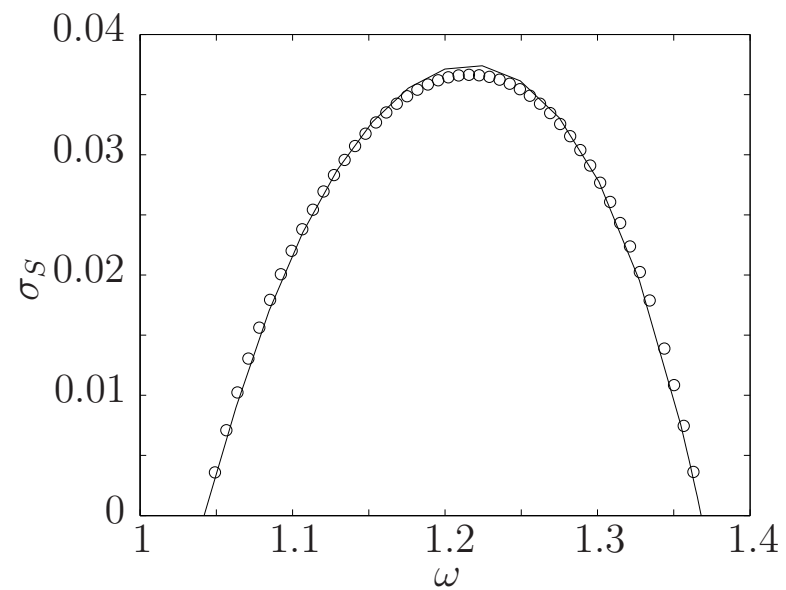

(b)

Figure 3. Comparison of the temporal ( $\circ)$ and spatial ( - ) linear stability analysis for the flow conditions and Blasius boundary layer profile in figure 2; $(a)$ streamwise wavenumber; (b) spatial growth rate. Here the Gaster transform of the temporal analysis is used

Additionally, the mode with the largest temporal growth rate is identified, for comparison with the DNS results in the next section. For the compressible Blasius profile with temperature-dependent viscosity at $R e_{\delta^{*}}=4200$, this most unstable mode has a wavelength $\lambda / \delta^{*}=2 \pi / \alpha \delta^{*}=4.70$, a frequency $\omega \delta^{*} / U=1.21$ and a growth rate $\sigma_{T} \delta^{*} / U=0.0272$ (see figure 4 and $3(a))$

\section{Direct numerical simulations}

\section{A. Numerical methods}

To perform simulations, we use our existing high fidelity computational aeroacoustic code that solves the fully two- and three-dimensional Navier-Stokes (NS) equations in Cartesian block-structured geometries. The method utilizes 6th-order-accurate compact finite difference schemes in the streamwise $\left(x^{-}\right)$and normal $(y-)$ directions, and explicitly 4th-order Runge-Kutta time advancement. When present, the spanwise $(z-)$ direction is supposed 


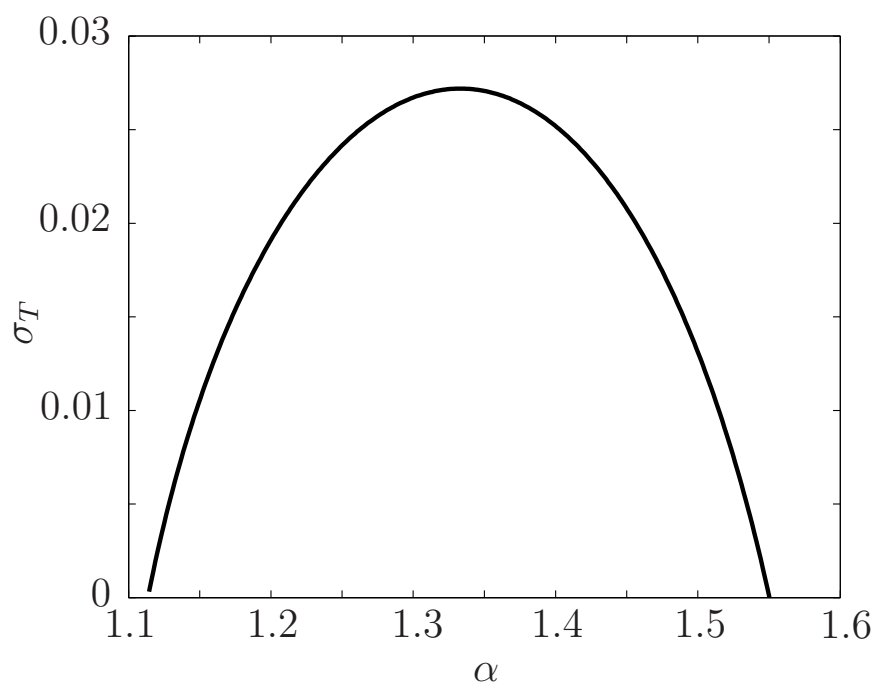

Figure 4. Growth rate from the theoretical temporal analysis, for the flow conditions and Blasius boundary layer profile in figure 2 .

homogeneous, and the derivatives are computed using Fourier-spectral differentiation. Optional boundary conditions include accurate inflow/outflow, nonreflecting, isothermal and adiabatic walls, as well as periodic and symmetry conditions. The code is fully parallelized using MPI, and a linearized version of the NS equations is also implemented. The algorithm introduces very little numerical dissipation and is able to efficiently resolve complex, unsteady flow physics, including acoustic wave generation and propagation. The algorithm and code has been developed, validated, and successfully implemented in studies of sound radiation from mixing layers, jets, vortex rings, and flow/acoustic instabilities in flows over open cavities (see Refs. 8-10 for details).

The equations are solved for a perfect gas, with constant specific heat capacities, $\gamma=1.4$ and constant Prandtl number $\operatorname{Pr}=0.7$. The wall temperature ratio $T_{w}$ is assumed to be uniform and constant, in general different from the freestream temperature. In this preliminary work, we consider constant viscosity $\left(\mu / \mu_{\infty}=1\right)$ and conductivity. Temperature variation in these properties will be included in future work. Nevertheless, we show that the present results are qualitatively similar to measurements with temperature-dependent properties, and therefore relevant for UAC design.

\section{B. Boundary layer stability}

First we consider the temporal linear stability of supersonic boundary layers, to identify the streamwise wavelength of the most unstable second mode. The flow parameters are again chosen to match the conditions used in the previous section and in Ref.1, apart from the 
temperature dependence of viscosity. The Mach number is $M=6$, and the wall temperature is constant $T_{w} / T_{\infty}=1.4$. Since there are no cavities, the characteristic length scale is the displacement thickness $\delta^{*}$ of the mean flow, rather than a cavity depth $H$.

Periodic boundary conditions are used in the streamwise $(x-)$ direction, and the nominally laminar boundary layer spreads in time rather than streamwise position. Since there are no variations in the $x$-direction in this case, that dimension of the computational domain can be arbitrary and is chosen as small as possible (i.e., 6 points)

We initialize the computations with an error-function profile in streamwise velocity (i.e., the correct self-similar solution as $M \rightarrow 0$ ), uniform pressure and use the Crocco-Busemann relation to compute the initial temperature profile for the chosen wall temperature ratio. By contrast with incompressible flow, there is no exact solution for the temporally-evolving compressible boundary layer. However, the initialization we choose minimizes sharp initial transients due to viscous dissipation in the boundary layer and the concurrent generation of a small positive normal velocity. This feature is important in hypersonic flow computations since sharp initial transients can quickly develop into shocks.

The nonlinear simulations are then advanced in time until the boundary layer thickness satisfies $\delta / H=2$, and the corresponding displacement thickness is computed. The resulting boundary layer profile is then frozen and used as the mean flow, $\bar{q}(y)$, in linear simulations. As initial condition, an acoustic perturbation of given streamwise wavelength, $\lambda$, is added to $\bar{q}$ and the linearized Navier-Stokes equations are solved. The least damped (or most unstable) eigenmode and the corresponding eigenvalue $\Omega=\omega+i \sigma_{T}$ are then determined from the long-time linear response of the boundary layer. The nondimensionalized growth/damping rate $\sigma_{T} \delta^{*} / U$ and frequency $\omega \delta^{*} / U$ are computed in each case for a set a discrete spanwise wavelength.

Table 1. Parameters and linear stability for the numerical simulations of a temporally-evolving boundary layer over a flat plate. Only the most unstable mode (largest growth rate) is reported.

\begin{tabular}{lccccccccc}
\hline \hline Run & \multicolumn{4}{c}{ Parameter } & \multicolumn{7}{c}{ Linear Stability } \\
& $\delta^{*} / \delta$ & $M$ & $T_{w} / T_{\infty}$ & $R e_{\delta^{*}}$ & $\lambda / \delta$ & $\sigma_{T} \delta^{*} / U$ & $\omega \delta^{*} / U$ & $c / U$ & \\
\hline case 1 & 0.60 & 6 & 1.4 & 2380 & 2.5 & $5.02 \mathrm{e}-4$ & 1.34 & 0.898 & DNS \\
& & & & & 2.5 & $4.80 \mathrm{e}-4$ & 1.34 & 0.898 & ARPACK \\
case 2 & \multirow{2}{*}{0.60} & \multirow{2}{*}{6} & \multirow{2}{*}{1.4} & \multirow{2}{*}{4200} & 2.5 & $7.95 \mathrm{e}-3$ & 1.36 & 0.900 & DNS \\
& & & & & 2.5 & $7.95 \mathrm{e}-3$ & 1.36 & 0.900 & ARPACK \\
\hline \hline
\end{tabular}

The parameters considered are reported in table 1 and the linear stability of the com- 
pressible boundary layer with constant viscosity is shown is figure 5. For both Reynolds numbers considered, $R e_{\delta^{*}}=2380$ and $R e_{\delta^{*}}=4200$, the streamwise wavelength of the dominant mode is $\lambda / \delta=2.5$, which is consistent with the typical assumption that the most amplified second-mode wavelength is approximately twice the boundary-layer thickness. ${ }^{1,3}$ Similarly to the spatial stability analysis, the temporal growth rate increases with Reynolds number. In contrast, the frequency is independent of $R e_{\delta^{*}}$. The phase velocity of the most amplified mode is $c / U \approx 0.9$, again consistent with the experimental and numerical observations that the phase velocity tends to the boundary-layer edge velocity of the mean flow. Fedorov et $a l .{ }^{3}$ reported similar phase velocity values in both experiment $(c / U=0.896)$ and theoretical model (0.916).

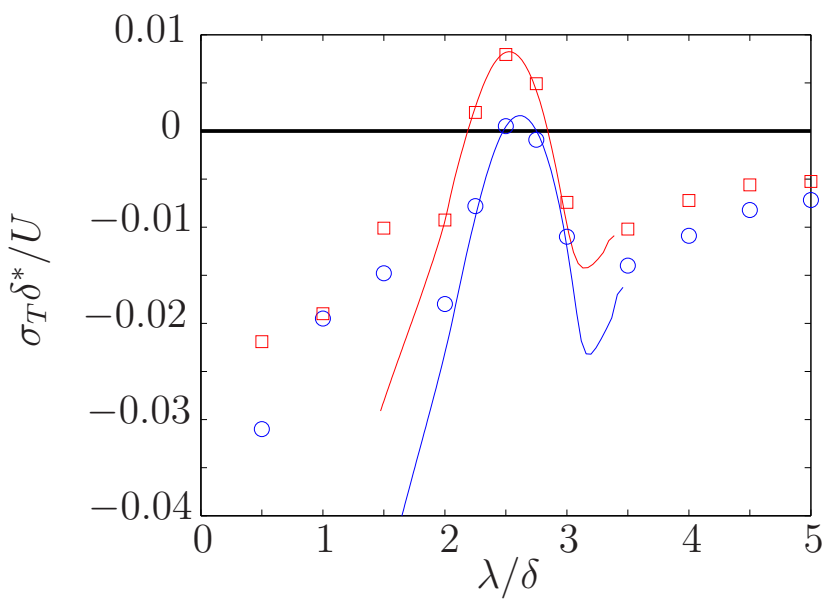

(a)

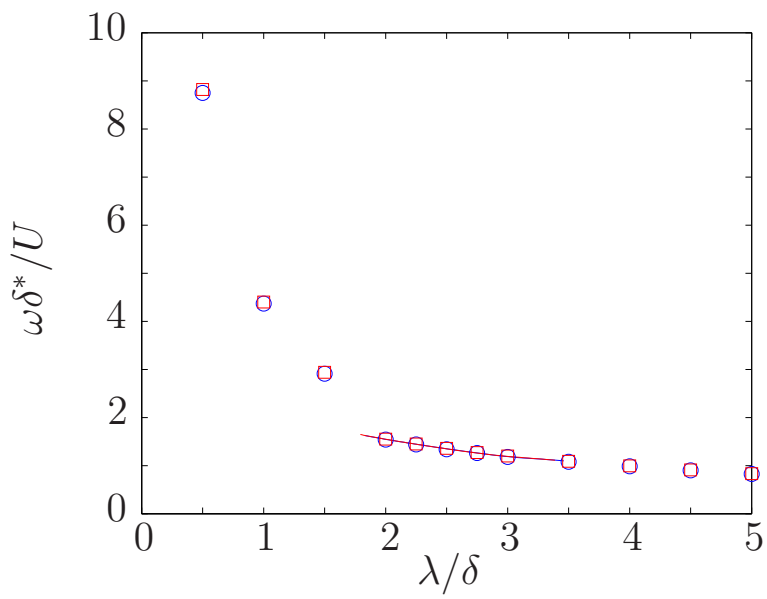

$(b)$

Figure 5. Temporal linear stability of the DNS boundary layer over a flat plate as a function of the streamwise wavelength of the disturbance, from DNS (symbols) and theoretical calculations (solid lines). Parameters: $M=6, T_{w} / T_{\infty}=1.4, R e_{\delta^{*}}=2380(\circ), R e_{\delta^{*}}=4200(\square)$; (a) temporal growth rate; $(b)$ frequency. The thick black line represents the stability transition $\sigma_{T}=0$

To validate the linear stability results, a direct approach is also considered, where the eigenmodes are directly searched for using an Arnoldi method developed in the ARPACK software, ${ }^{11}$ rather then isolated through long-time integration. As expected, the dominant eigenvalue computed with ARPACK is in excellent agreement with the results of the linear stability analysis (see table 1). Computations with ARPACK also confirmed that there is only one mode of positive growth rate at the considered wavelength.

The theoretical stability calculation are recomputed using the DNS velocity and temperature profiles (rather than the previous Blasius profiles). Here, the temporal analysis is conducted with constant viscosity, and the results are in excellent agreement with the DNS estimates, as shown in figure 5. For these conditions and $R e_{\delta^{*}}=4200$, the most unstable mode predicted from theory has a wavelength $\lambda / \delta=2.52$ (i.e., $\lambda / \delta^{*}=4.21$ ), with a 
frequency $\omega \delta^{*} / U=1.34$ and a growth rate $\sigma_{T} \delta^{*} / U=0.00824$.

We observe that the growth rate is underestimated compared to analysis for the Blasius profile with temperature-dependent viscosity reported in the previous section, as well as slight variation in wavelength and frequency. We identify the different mean flow (see figure 2) used in the two methods as the primary source of these discrepancies, rather the difference in viscosity. However, for the temporal stability of supersonic boundary layers over porous surface, the compressible Blasius solution cannot be used directly as mean flow for the linear analysis. In this case, DNS data is required to accurately capture the mean flow features in the vicinity of the pores. As our overall objective is to quantify the stabilizing effect of the UAC on the second-mode instability using a temporal analysis, our approach is the use DNS profiles consistently and quantify the differences introduced by these simplifications.

The temporal stability analysis also recovers the presence of the first and second modes identified by Fedorov and Tumin ${ }^{12}$ (also referred as mode $\mathrm{F}$ and $\mathrm{S}$ by Fedorov ${ }^{13}$ ). In their figure 7, the numerical evaluation of the temporal eigenvalues for the first and second modes are presented as a function of the streamwise wavenumber, for a compressible boundary layer over an adiabatic wall at $M=5.6$ and $R e=1219.5$. The length scale in their work is $L^{*}$ such that the boundary layer thickness verifies $\delta / L^{*} \approx 10$. The first mode (or $\mathrm{F}$ for Fast) has a phase speed of $1+1 / M$ as $\alpha$ goes to zero, while the second mode (or $\mathrm{S}$ for Slow) phase speed tends to $1-1 / M$. As the streamwise wavenumber of the instability is increased, they report that the temporal growth rate of the first mode decays, while the second mode becomes unstable. The most amplified wavenumber is $\alpha L^{*} \approx 0.25$, which leads to $\lambda / \delta \approx 2.51$, compare to $\lambda / \delta \approx 2.5$ for our temporal stability analysis. As shown in figure 6 , the frequency and most unstable wavenumber again agree between the two analyses.

\section{Linear computations demonstrating the stabilizing effect of the UAC}

Now that the streamwise wavelength of the most unstable second mode (i.e., $\lambda / \delta=2.5$ ) is identified for the chosen flow conditions, we perform two-dimensional DNS of the boundary layer with and without the UAC for a periodic domain of streamwise extent equal to an integer number of that wavelength.

The porous coating considered here corresponds to the 2-D micro-cavities used in Ref. 7 to study the acoustic scattering properties of UAC. That is, the pores have a constant length to depth ratio $A r=2 b / H=0.12$ and the porosity is $\phi=2 b / s=0.48$, where $s$ is the spacing between cavities. For this configuration with the pores, the mesh contains about two hundred thousand grid points, with 12 and 100 points across each cavity length and depth, respectively.

All the simulations are performed following the same procedure that the boundary layer computations, and on the same Cartesian grid, with the pores extending to $y / H=-1$ under 


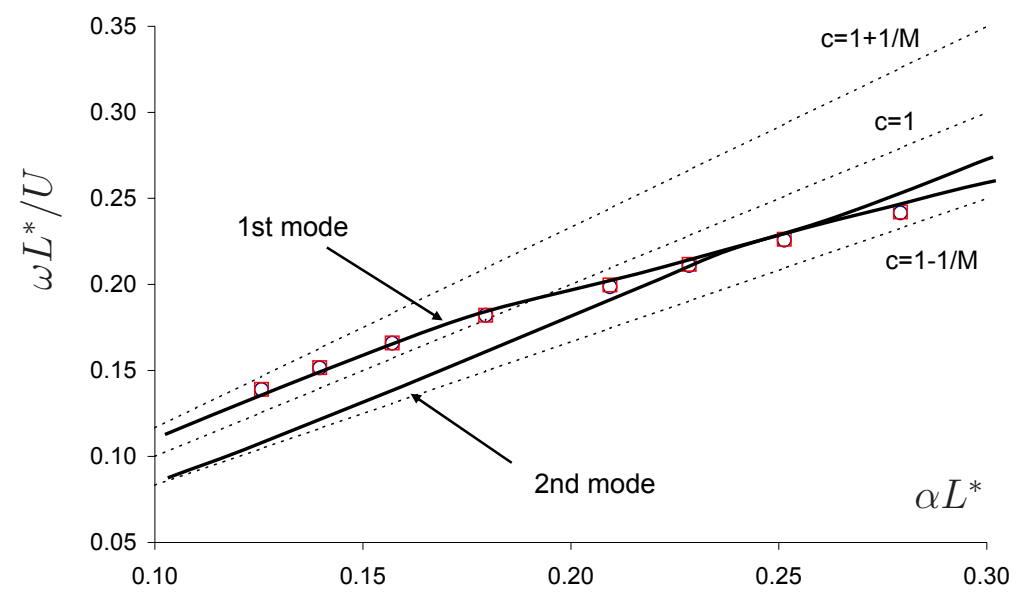

Figure 6. Frequency as a function of the streamwise wavenumber for comparison with figure 7 in article by Fedorov and $\operatorname{Tumin}^{\mathbf{1 2}}\left(-\right.$ ): $M=6, T_{w} / T_{\infty}=1.4,(\circ) R_{\delta^{*}}=2380,(\square)$ $R e_{\delta^{*}}=4200$. Only the most unstable mode is shown. The 1st and 2nd mode identified by Fedorov and Tumin ${ }^{12}$ are reported.

to solid surface at $y=0$. The dimension of the computational domain in the streamwise (periodic) direction is $\Lambda=5 \mathrm{H}$. As the nonlinear simulations are again advanced in time until $\delta / H=2$, The streamwise extent of the domain is $\Lambda / \delta=2.5$, equal to one time the wavelength of the most unstable second-mode. Typical UAC are designed with many pores per instability wavelength, $N=\lambda / s$, to avoid resonant interactions between the boundary layer disturbance and near-wall perturbations induced by the coating structure as well as roughness effect. For the aforementioned parameters and porosity, this condition is satisfied, since $N=20$. For the boundary layer over the porous coating, the layer properties are computed over the top solid wall, rather than above a cavity, and the displacement thickness is again $\delta^{*} / \delta \approx 0.60$

While the boundary layer profile is unchanged for the flat plate and only a function of the normal direction, the mean (frozen) flow field with UAC is two-dimensional, as the flow at the cavity mouths is accounted for (see figure 7). We then perform linear simulation about these mean flow, with a circular acoustic pulse of Gaussian shape is added as initial condition. Because of the general form of the perturbations, several modes are initially excited, leading to some transient. Eventually, the least damped (or most unstable) eigenmode and the corresponding eigenvalue is determined from the long-time linear behavior of the flow.

Figure 8 shows the time-history of the perturbation velocity $u^{\prime} / U$ for the boundary layer at $M=6, T_{w} / T_{\infty}=1.4$, and $R e_{\delta^{*}}=4200$, above the flat plate and over the porous coating. The probe is located at $(x / H, y / H)=(1,0.1)$, and similar results are obtained for different locations and flow variables. As predicted by the linear analysis of the boundary layer stability presented in the previous section, exponential growth of the perturbation 


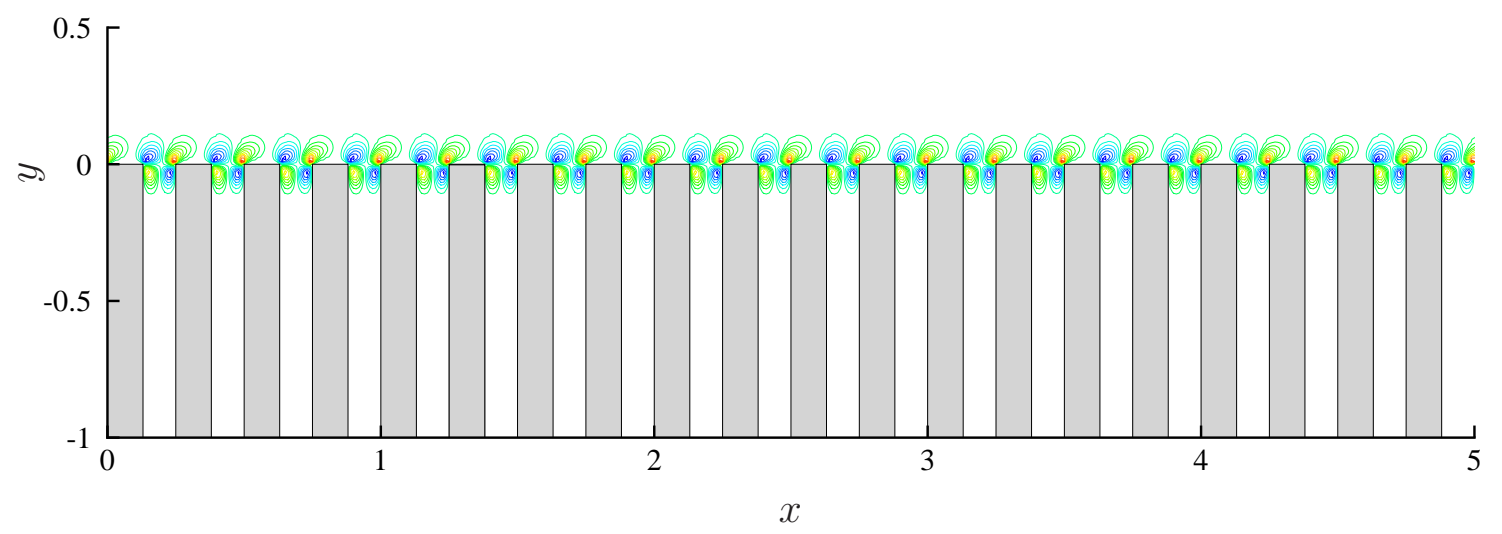

Figure 7. Normal velocity contours for the mean (frozen) flow of the boundary layer profile over the porous coating. Fifteen contours between $\bar{v} / U=-0.0025$ and 0.0035 are shown.

is observed at large time. The wavelength of the instability is $\lambda / \delta=2.5$, the estimated frequency $\omega \delta^{*} / U=1.36$ and growth rate $\sigma_{T} \delta^{*} / U=0.00793$ match the previous results. In contrast, the instability is completely stabilized by the porous coating.

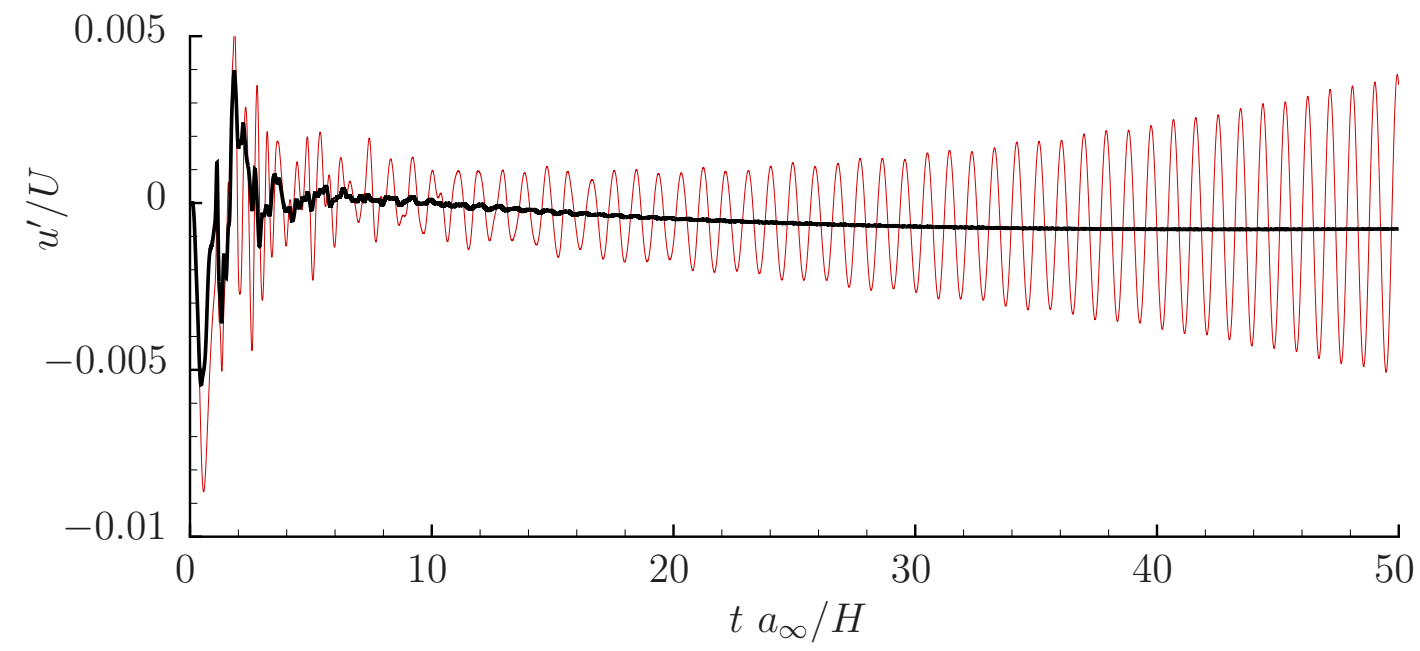

Figure 8. Time-history of the perturbation velocity $u^{\prime} / U$ with $(-)$ and without $(-$ ) the porous coating

To confirm this result, the theoretical stability calculation using the DNS velocity and temperature profiles (and constant viscosity) are performed with an artificial boundary condition to take into account the presence of the UAC. The boundary conditions on the porous wall are calculated using the theoretical model from Ref. 1 and the solutions for the propagation of acoustic disturbances within a slot. ${ }^{14}$ The model assumptions are discussed in details in Ref. 7. The calculations were carried out for different porosity $0 \leq \phi \leq 0.8$, while 
the disturbance wavelength is fixed at $\lambda / \delta=2.5$. For these conditions, the growth rate of the most unstable second mode decays rapidly with increasing porosity (see figure 9), such that the instability is stable for $\phi>0.06$. At the porosity $\phi=0.48$ considered in the DNS, the theoretical calculation predicts a decay rate $\sigma_{T} \delta^{*} / U=-0.06$. Therefore, the perturbation in the DNS should be damped quickly, which is what is observed in the simulations.

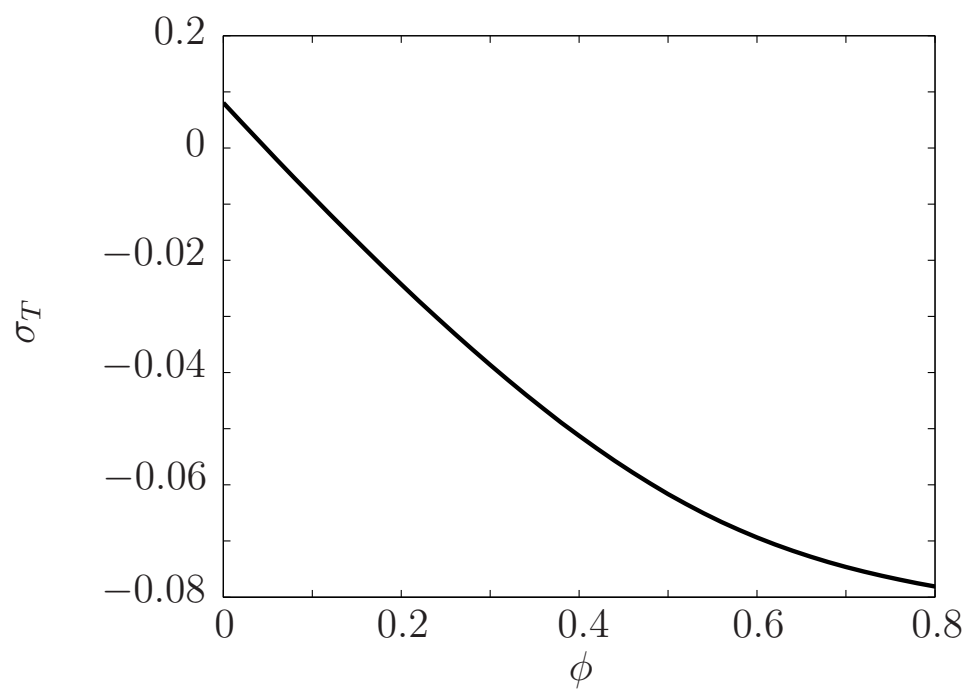

Figure 9. Growth rate from the theoretical temporal analysis with artificial porous boundary conditions, for the DNS profile in figure 2

\section{Conclusions}

In this paper, we have attempted to define computationally tractable model problems that account for the detailed flow physics associated with micro-cavities that attenuate secondmode instabilities in hypersonic boundary layers. We have provided evidence that it is sufficient for many purposes to consider temporally (rather than spatially) evolving boundary layers and instabilities. This greatly reduces computational expense, since it allows the computational domain to include a single wavelength of the instability (which still may require about 20 micro-cavities in the simulation).

In order to verify that the physics are not substantially altered by the assumption of streamwise periodicity, we revisited linear stability analysis and showed that second mode instability has a sufficiently slow streamwise growth to allow spatial instability results to be accurately recovered from the temporal ones via the Gaster transformation, provided the mean velocity and temperature profiles are the same in both cases.

We then used the corresponding temporal instability calculations (for a flat plate without UAC) to validate linearized DNS computations of the same problem. We used high- 
order-accurate non-dissipative numerics to resolve the hypersonic shock-free flow. DNS and instability calculations are in excellent agreement. As compared to the spatial problem, there are differences in the results not accounted for in the Gaster transformation, since the mean velocity and temperature profiles are somewhat different for spatially and temporally growing boundary layers. For simplicity, we also used constant viscosity in the DNS; profiles associated with temperature-dependent properties differ and also lead to different stability frequencies and growth rates. However, within the framework of these simplifications, linear DNS of plates with UAC were successful at damping second-mode instabilities by an amount consistent with approximate theory and experiments for the given Mach number, Reynolds number, and UAC parameters.

In future work, we will relax the constant viscosity assumption, and perform detailed analysis of linear and nonlinear second-mode attenuation by UAC within the temporally evolving framework. In particular, we will investigate the details of instability-wave absorption by the micro-cavities, and validate (and perhaps suggest improvements to) approximate porous-wall boundary conditions for engineering predictions of UAC performance.

\section{Acknowledgments}

The authors would like to acknowledge contributions to this work by Norm Malmuth, whose passionate work on diverse topics, including UAC, and generosity to his collaborators will be sorely missed. This work was supported by the Air Force of Scientific Research under Contract FA9550-06-C-0097.

\section{References}

${ }^{1}$ Fedorov, A. V., Malmuth, N. D., Rasheed, A., and Hornung, H. G., "Stabilization of hypersonic boundary layers by porous coatings," AIAA Journal, Vol. 39(4), 2001, pp. 605-610.

${ }^{2}$ Rasheed, A., Hornung, H. G., Fedorov, A. V., and Malmuth, N. D., "Experiments on passive hypervelocity boundary layer control using an ultrasonically absorptive surface," AIAA Journal, Vol. 40(3), 2002, pp. 481-489.

${ }^{3}$ Fedorov, A. V., Shiplyuk, A. N., Maslov, A. A., Burov, E. V., and Malmuth, N. D., "Stabilization of a hypersonic boundary layer using and ultrasonically absorptive coating," Journal of Fluid Mechanics, Vol. 479, 2003, pp. 99-124.

${ }^{4}$ Fedorov, A. V., Kozlov, V. F., Shiplyuk, A. N., Maslov, A. A., Sidorenko, A. A., Burov, E. V., and Malmuth, N. D., "Stability of hypersonic boundary layer on porous wall with regular microstructure," AIAA Paper 2003-4147, 2003.

${ }^{5}$ Bountin, D., Shiplyuk, A., Maslov, A., and Chokani, N., "Nonlinear aspects of hypersonic boundary layer stability on a porous surface," AIAA Paper 2004-0255, 2004.

${ }^{6}$ Maslov, A. A., Shiplyuk, A. N., Sidorenko, A. A., Polivanov, P., Fedorov, A. V., Kozlov, V. F., and 
Malmuth, N. D., "Hypersonic laminar flow control using a porous coating of random microstructure," AIAA Paper 2006-1112, 2006.

${ }^{7}$ Brès, G. A., Colonius, T., and Fedorov, A. V., "Interaction of acoustic disturbances with micro-cavities for ultrasonic absorptive coatings," AIAA Paper 2008-3903, 2008.

${ }^{8}$ Colonius, T., "Modeling artificial boundary conditions for compressible flow," Annual Review of Fluid Mechanics, Vol. 36, 2004, pp. 315-345.

${ }^{9}$ Colonius, T. and Lele, S., "Computational Aeroacoustics: Progress in Nonlinear Problems of Sound Generation," Progress in Aerospace Sciences, Vol. 40, No. 6, 2004, pp. 345-416.

${ }^{10}$ Brès, G. A. and Colonius, T., "Three-dimensional instabilities in compressible flow over open cavities," Journal of Fluid Mechanics, Vol. 599, 2008, pp. 309-339.

${ }^{11}$ Lehoucq, R., Maschhoff, K., Sorensen, D., and Yang, C., "ARPACK software. Website: http://www.caam.rice.edu/software/ARPACK/," 2007.

${ }^{12}$ Fedorov, A. V. and Tumin, A., "Initial-value problem for hypersonic boundary-layer flows," AIAA Journal, Vol. 41(3), 2003, pp. 379-389.

${ }^{13}$ Fedorov, A. V., "Receptivity of a high-speed boundary layer to acoustic disturbances," Journal of Fluid Mechanics, Vol. 491, 2003, pp. 101-129.

${ }^{14}$ Kozlov, V. F., Fedorov, A. V., and Malmuth, N. D., "Acoustic properties of rarefied gases insides pores of simple geometries," Journal of the Acoustic Society of America, Vol. 117(6), 2005, pp. 3402-3412. 